\title{
Monitoring magnesium efflux cyclic AMP-induced in HL60 cells by using a new hydroxyquinoline fluorescent chemosensor
}

\author{
Chiara Marraccini, ${ }^{1}$ Azzurra Sargenti, ${ }^{2}$ Lucia Merolle, ${ }^{2}$ Concettina Cappadone, ${ }^{2}$ \\ Giovanna Farruggia, 2,3 Stefano lotti ${ }^{2,3}$ \\ ${ }^{1}$ Department of Life Sciences, University of Modena and Reggio Emilia; ${ }^{2}$ Department of Pharmacy \\ and Biotechnologies, University of Bologna; ${ }^{3}$ National Institute of Biostructures and Biosystems, \\ Roma, Italy
}

\begin{abstract}
Cellular homeostasis of magnesium is still unclear. Several studies documented the occurrence of fluxes of magnesium across the plasmamembrane within minutes from the application of metabolic or hormonal stimuli. These fluxes, however, result in limited variation of free $\mathrm{Mg}^{2+}$ intracellular concentration and large changes in total Mg content. It has been reported that a stimulation with cyclic AMP caused a movement of total magnesium within 10 min after treatment in cardiomyocytes. In this study we tested this hypothesis in HL60 leukemic cells, not excitable but highly proliferating cell model. We evaluated Mg flux by DCHQ5, the phenyl-derivative of hydroxyquinoline fluorescent probe family. We observed a drastic decrease of intracellular total magnesium in the first $3 \mathrm{~min}$. We also verified that at least $10 \%$ of the total intracellular amount of magnesium moved in the supernatant of stimulated cells.
\end{abstract}

\section{Introduction}

Cellular magnesium homeostasis is still unclear, although this cation is essential for numerous cell functions. ${ }^{1,2}$ Several studies documented the occurrence of fluxes of magnesium across the plasmamembrane within minutes from the application of metabolic or hormonal stimuli. These fluxes, however, result in limited variation of free $\mathrm{Mg}^{2+}$ intracellular concentration and large changes in total $\mathrm{Mg}$ content. ${ }^{3} \mathrm{DCHQ} 5$ probe, which is stable up to $30 \mathrm{~min}$ of incubation and

Correspondence: Giovanna Farruggia, Department of Pharmacy and Biotechnologies, University of Bologna, via Belmeloro 6, 40126 Bologna, Italy. Tel/Fax: +39.051.2099700.

E-mail: giovanna.farruggia@unibo.it

Key words: magnesium efflux, cAMP, hydroxyquinoline derivative.

(C) Copyright C. Marraccini et al., 2014

Licensee PAGEPress, Italy

Journal of Biological Research 2014; 87:2137

doi:10.4081/jbr.2014.2137

This article is distributed under the terms of the Creative Commons Attribution Noncommercial License (by-nc 3.0) which permits any noncommercial use, distribution, and reproduction in any medium, provided the original author(s) and source are credited. highly retained within loaded cells, results to be the proper tool for monitoring magnesium content. ${ }^{4}$

It has been reported that a stimulation with cyclic AMP (cAMP) caused a movement of total magnesium within 10 min after treatment in cardiomycites, suggesting that this stimulus caused a magnesium efflux in excitable cells. ${ }^{5}$ In this study we tested this hypothesis in HL60 leukemic cells, a not excitable but highly proliferating cell model. We evaluated Mg flux by DCHQ5 using spectrofluorimetric and cytofluorimetric assays.

\section{Materials and Methods}

HL60 cells were maintained in Roswell Park Memorial Institute (RPMI) 1640 medium supplemented with $2 \mathrm{mM}$ L-Glutamine, 10\% foetal bovine serum (FBS) at $37^{\circ} \mathrm{C}$ and $5 \% \mathrm{CO} 2$. Total magnesium was assessed incubating $5 \times 10^{5}$ cells/mL in phosphate buffered saline (PBS) without $\mathrm{Mg}^{2+}$ with DCHQ5 $15 \mu \mathrm{M}$ for 15 min in the dark. Then cells were treated with different concentration of cAMP and analyzed by cytofluorimetric and spectrofluorimetric assay. Magnesium efflux was evaluated treating $3 \times 10^{6}$ cells/mL in PBS without $\mathrm{Mg}^{2+}$ for $3 \mathrm{~min}$ with cAMP. The cells were then centrifugated and the magnesium in the supernatant was measured by DCHQ5 probe using a calibration curve. Intracellular free $\mathrm{Mg}^{2+}$ were determined by Mag-Fluo4-AM staining: HL60 cells were incubated with $0.8 \mathrm{M}$ fluorophore in medium without $\mathrm{Mg}^{2+}$ for $30 \mathrm{~min}$ at $37^{\circ} \mathrm{C}$ and analyzed by flow cytometry. Dead cells were excluded by propidium iodide (PI) incorporation.

\section{Results}

We evaluated Mg flux by using DCHQ5 spectrofluorimetric assay: the cells were treated with different cAMP concentrations and the fluorescence intensity was monitored for 10 min after the stimulation (Figure 1). A marked dose-dependent decrease of total intracellular magnesium was observed in the first 3 min after treatment; moreover, this effect continued in the next time of analysis, confirming that cAMP induced an extracellular Mg efflux.

We then quantified the intracellular magnesium content released in the supernatant after $3 \mathrm{~min}$ of $1.25 \mathrm{mM}$ cAMP stimulation by DCHQ5: we found that magnesium in cAMP-treated cells is doubled respect to the physiological release observed in control cells (Figure 2). When we compared the magnesium released $\left(5.3 \mathrm{nmol} / 10^{6}\right.$ cells $)$ with the normal intracellular content of this cation $\left(30 \mathrm{nmol} / 10^{6}\right.$ cells $)$, we found that $1.25 \mathrm{mM}$ cAMP stimulation released about $20 \%$ of this cation in the extracellular medium.

We also verified that the observed magnesium release was not due 

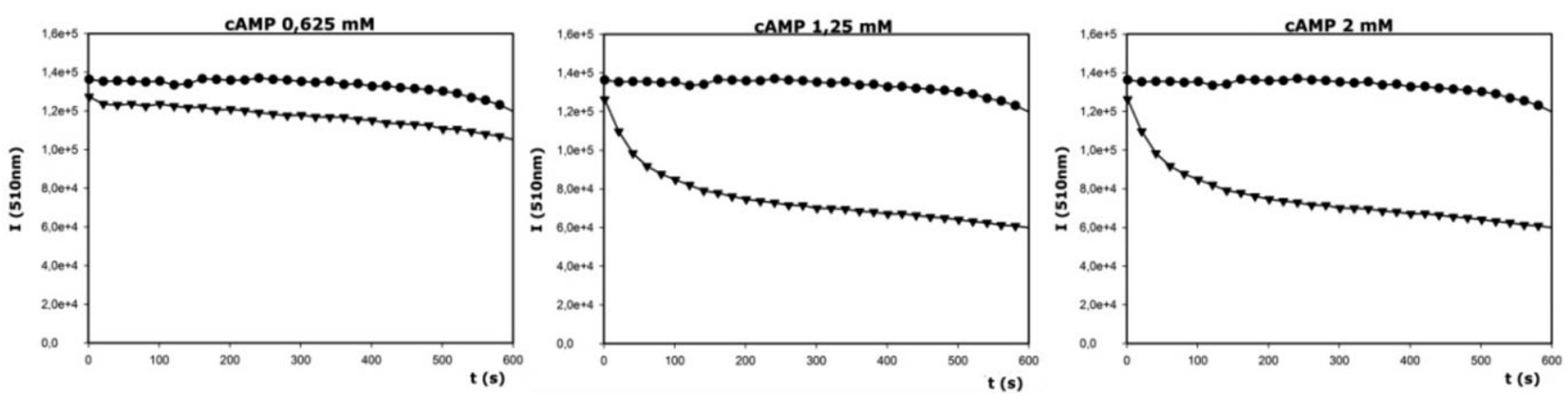

Figure 1. Time course of DCHQ5 fluorescence intensity $\left(\lambda_{\text {ex }} 360 \mathrm{~nm}, \lambda_{\mathrm{em}} 510 \mathrm{~nm}\right)$, in stimulated $(\nabla)$ and control cells $(\odot)$.

to cellular swelling, as shown by the vitality assay reported in Figure 3 , where the plots of the fluorescence of DCHQ5 in function of PI fluorescence in treated and control samples are reported.

It is reported that in cardiomyocytes cAMP stimuli caused a variation in total intracellular magnesium but not in the free fraction: in HL60 cells, the analysis of free, ionized magnesium by Mag-Fluo-4 probe $^{3}$ did not show any difference between control cells and cells treated with cAMP, even at the higher dose (data not shown).

\section{Discussion}

We demonstrated that cAMP stimulation promotes a magnesium release also in highly proliferating cancer cells and not only in excitable cells. The results obtained suggest a possible role for magnesium as second messenger as proposed in the past on theoretical basis. ${ }^{2}$ Moreover, our results confirmed that DCHQ5 is a suitable tool to deepen our knowledge on magnesium homeostasis, as it can be used for the quantification of total intracellular magnesium, as well as for monitoring magnesium cellular fluxes.

\section{References}

1. Wolf FI, Cittadini A. Magnesium in cell proliferation and differentiation. Front Biosci 1999;4:607-17.

2. Rubin H. Magnesium: the missing element in molecular views of cell proliferation control. Bioessays 2005;27:311-20.

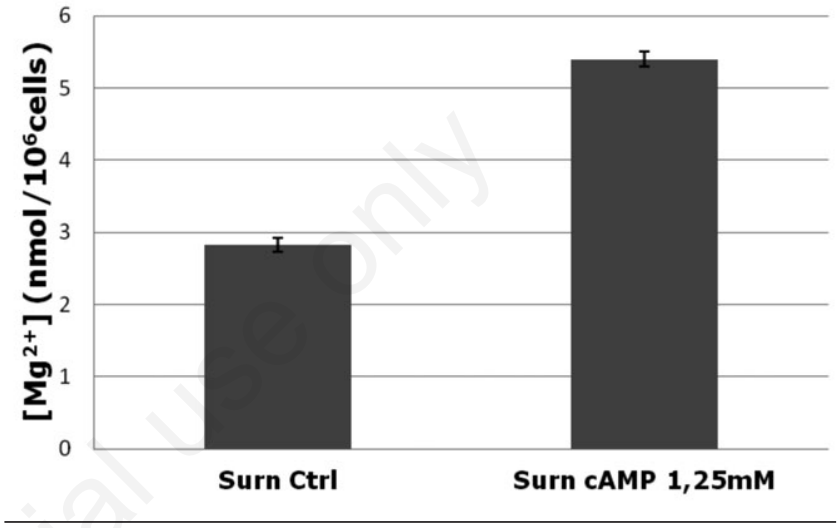

Figure 2. Quantification by DCHQ5 of the intracellular magnesium content released in the supernatant after $3 \mathrm{~min}$ of $1.25 \mathrm{mM}$ cyclic AMP stimulation.

3. Romani AM. Cellular magnesium homeostasis. Arch Biochem Biophys 2011;512:1-23.

4. Marraccini C, Farruggia G, Lombardo M, et al. Diaza-18-crown-6 hydroxyquinoline derivatives as flexible tools for the assessment and imaging of total intracellular magnesium. Chem Sci 2011;3:727-34.

5. Fatholahi M, Lanoue K, Romani A, et al. Relationship between total and free cellular $\mathrm{Mg}^{2+}$ during metabolic stimulation of rat cardiac muocytes and perfused hearts. Arch Biochem Biophys 2000;374:395-401.
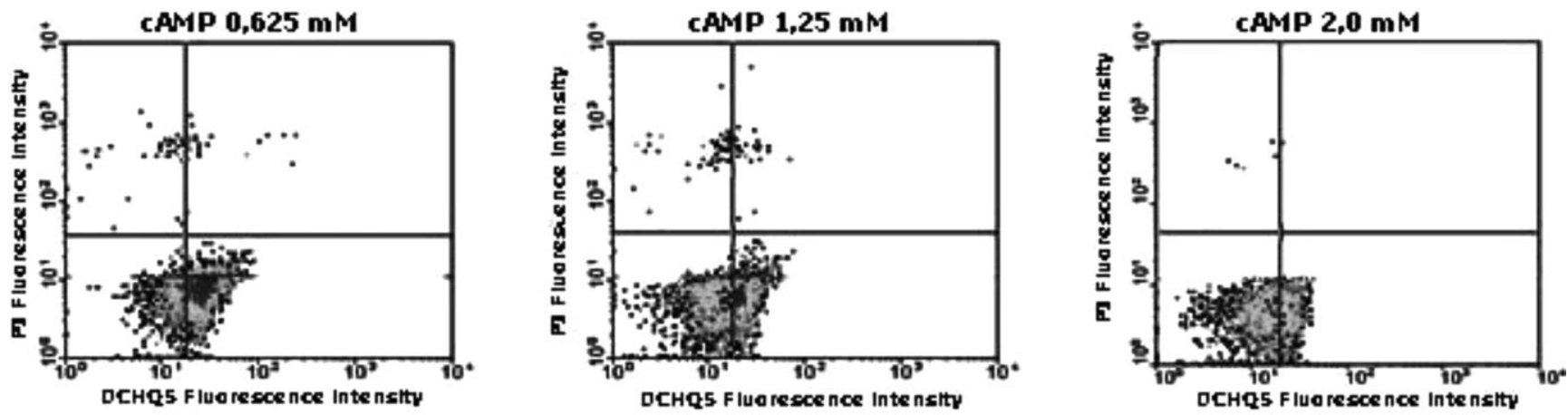

Figure 3. Cytofluorimetric vitality assay with propidium iodide: the samples, treated with cyclic AMP, were stained with DCHQ5 and propidium iodide: damaged, propidium iodide-positive cells fall in the upper quadrants. 